\title{
Allocation of Distributed Generations Based on TSPSO Algorithm
}

\author{
Liu Wei, Zhang Haiyan and Zhang Xu \\ School of Electrical Information Engineering, Northeast Petroleum University \\ Daqing, Heilongjiang, China \\ nepuliuwei@163.com,happyswimfly@163.com,xiaorenwu_est@163.com
}

\begin{abstract}
When the system is with DG (distributed generation), Power system structure has changed The structure has change to complicated new model with distributed generations from traditional open network. The voltage and power losses of traditional network will be influenced by the location connected with $D G$, reactive power, active power and the number of DG. The purpose to connect DG is to improve reliability of the system, reduce the loss of network and reduce the cost. In order to achieve this goal, this paper analyzes the indicator of power loss and takes it as objective function. Considering the superior properties of particle swarm optimization algorithm in solving discrete values problem, the algorithm is improved by the tabu search mechanism, we use the TSPSO (Tabu Search mechanism Particle Swarm Optimization) algorithm to study the problems which include the positions, capacity and numbers of DGs. At last, verify the validity of the method by simulation experiment.
\end{abstract}

Keywords: TSPSO; DG; power loss; voltage profile

\section{Introduction}

In modern society, the deficiency of centralized power increasingly exposed such as electricity crisis, energy crisis and large-scale power outage. These problems show electric power system with a single power supply cannot meet the requirement of system and users as the requirement of power supply quality and reliability is increasing day by day. It's main operation mode is large scale power grids, a lot of generating set and high voltage, etc. In this environment, Distributed generation technology arised. Many and various types of DGs connect to different power network. This has become an important direction of the electric power industry development in the future [1].But the DG connected to power network may produce influence in many aspects. Different position and capacity may produce the change of stability, economy, electric power loss and reliability. In the development of future electric power industry, optimal configuration of DG included positions and capacity to connect becomes particularly important.

The optimization problems of DG in distribution network due to the different types of decision variables are divided into two categories, namely: 1) Single planning approaches; 2) Integrated and coordinated planning approaches. The former is to optimize capacity and locations of DGs in the situation which feeder substation configuration and system is unchanged. The latter is a planning in the whole distribution network for the coordination and optimization, there are many types of decision variables, so the latter is the whole planning targeted at distribution stations and substations, distributed power, or feeder, etc. The related research: Literature [2] proposes a multi-objective optimization algorithm of Pareto 
Computing, which is called SPEA (the Evolutionary Algorithm of Pareto), and apply it to different distributed network planning issues. But Injeti and others proposed an optimized planning and a method which is running with distributed power networks, and uses simulations to give verification in different node networks. In [3], Authors propose the method which uses genetic algorithm to optimize the location of distributed power, and also study fuzzy control theory and PSO Algorithm to resolve the installing DGs problem in a radial network, and after that, the authors study the impact on various parameters [ 4-6 ].

In distributed power system with distributed power among the various studies over the years proved connect distributed generations have an impact on the entire system, as long as a reasonable number of distributed generations, positions and optimize the allocation of capacity, and simulation results validate the electricity network will have a positive effect, improve level power quality of system, system reliability and so on. This paper proposes the method of improved particle swarm optimization indepth discussion, and using power loss as an objective function to analyze and research the distributed generations optimization, put forward a new theoretical ideas at the same time, verified the advantages of algorithm.

\section{Research and Analysis of TSPSO Algorithm}

The problem of power system with distributed power supply network involved in the optimization is a large complex optimization problem, so the requirement of solving this type of problems seems relatively high, not only the convergence accuracy is required high, but also requires the convergence faster.

\subsection{Research of TSPSO algorithm}

In this study, combine with the advantages and disadvantages of various algorithms, selection algorithm based on particle swarm optimization using tabu search mechanism to improve the initially algorithm, so that achieve complementary advantages, improve the algorithm optimization ability, accelerate the convergence speed of improved intelligent optimization algorithms, then validate and analyze, and ultimately its application to practical problems which guide the selection of optimal solutions.

Find the optimal solution, the particles used to update the velocity and position of the formula is as follows:

$$
\begin{gathered}
v_{k+1}=c_{0} v_{k}+c_{1}\left(\text { pbest }_{k}-x_{k}\right)+c_{2}\left(\text { gbest }_{k}-x_{k}\right) \\
x_{k+1}=x_{k}+v_{k+1}
\end{gathered}
$$

Where, $v_{k}$ is the particle flying velocity vector, $x_{k}$ is current particle position, pbest $_{k}$ the position of the particle current optimal solution, gbest $_{k}$ is the current optimal position of the whole population, $\mathrm{c} 0, \mathrm{c} 1, \mathrm{c} 2$ are cognitive factors.

Added in distributed network, the number, positions and size of distributed generations are discrete values. Therefore, the proposed method requires the ability to solve discrete optimization problems has a strong advantage, and PSO in solving issues such as discontinuous, nondifferentiable, nonlinear combinatorial optimization problems shows outstanding advantages, so this article selected PSO algorithm. However, PSO is also flawed, for example: local optimum conditions and prone to premature phenomenon, in this paper introduces a new mechanism for particle swarm algorithm to solve this problem.

Tabu search (TS) algorithm has the following advantages: a strong "climbing" capability, the search process appears acceptable inferior solutions, and it could jump out of local 
optima. So you can take advantage of this feature of TS search mechanism to improve particle swarm algorithm. In the extension of the advantages of the original algorithm, and improve it.

Specific processes are showed in Figure 1.

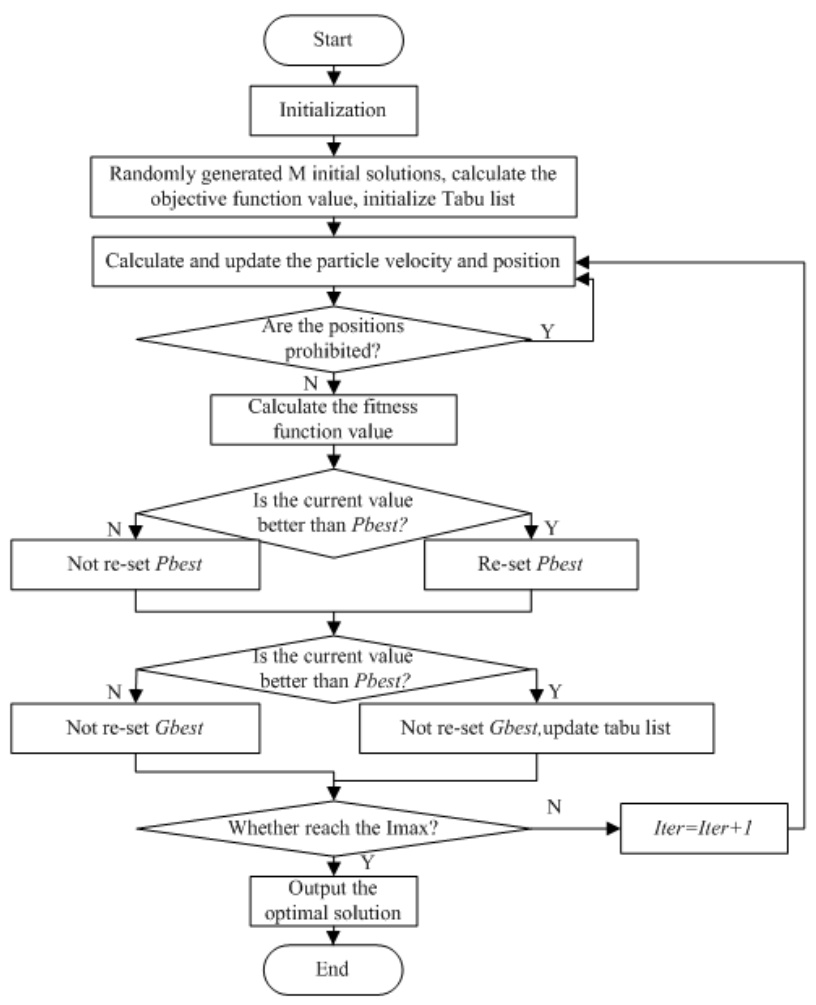

\section{Figure 1. Overall process in the proposed method}

The steps of TSPSO algorithm as follows:

1. Initialization of the parameter data, etc. Setting the initial population size G, tabu list length $\mathrm{L}$, the maximum number of iterations $\mathrm{I}_{\max }$, and the control parameter $\mathrm{c} 0$, the upper and lower limits of $\mathrm{c} 1, \mathrm{c} 2$.

2. Randomly generate a set of solutions, calculate the objective function value. Determine the optimal solution of the population $G_{b e s t}$, and sends the value to tabu objects, term is L. Set the current position of each particle is the current optimal solution $P_{\text {best }}$, set the initial number of iterations Iter $=1$.

3. Use equations (1) and (2) to update the position and velocity of particles.

4. Check whether the position of the particle banned, and if so, recalculate the flight speed and position of the particle; if not, continue to the next process.

5. Computing the individual objective function value obtained, and comparing it to $f_{\text {ibest }}$ which is the corresponding target value of $P_{i}$, after that we determine the global optimal solution.

6. Determine whether the number of iterations has been reached maximum Imax, if has been reached, then the optimum output groups $G_{b e s t}$, if it is not reached, then set, go to step 3 . 


\subsection{Analysis of TSPSO algorithm test results}

The present study may use 5 typical characteristics of test simulation function judgment and analytical improvements to the TSPSO algorithm. And then test results with standard comparison of particle swarm optimization, genetic algorithm, the algorithm is used to validate the rationality, effectiveness, as well as good optimization skills.

The algorithm to select test function:

1. Spherical function:

$$
f_{1}=\sum_{i=1}^{n} x_{i}^{2} \quad\left(-100 \leq x_{i} \leq 100\right)
$$

2. Rosenbrock function:

$$
f_{2}=\sum_{i=1}^{n-1}\left(100\left(x_{i+1}-x^{2}\right)^{2}+\left(x_{i}-1\right)^{2}\right)\left(-100 \leq x_{i} \leq 100\right)
$$

3. Griewank function:

$$
f_{3}(x)=\frac{1}{4000} \sum_{i=1}^{n} x_{i}^{2}-\prod_{i=1}^{n} \cos \left(\frac{x_{i}}{\sqrt{i}}\right)+1 \quad\left(-600 \leq x_{i} \leq 600\right)
$$

4. Rastrigin function:

$$
f_{4}(x)=\sum_{i=1}^{n}\left(x_{i}^{2}-10 \cos \left(2 \pi x_{i}\right)+10\right)\left(-10 \leq x_{i} \leq 10\right)
$$

5. Schaffer function:

$$
f_{3}=\frac{\sin ^{2} \sqrt{x_{1}^{2}+x_{2}^{2}}-0.5}{\left[1+0.001\left(x_{1}^{2}+x_{2}^{2}\right)\right]^{2}}-0.5 \quad\left(-100 \leq x_{i} \leq 100\right)
$$

Spherical function $f_{1}$ is a unimodal functions of spherical model, you can use it to verify the characteristics of local optimization algorithm, the global minimum point is $x_{i}=0(i=1,2, \ldots \mathrm{n})$. Rosenbrock function $f_{2}$ is a quadratic function of morbid, and characteristics of the function is difficult to minimize. Although it is easier to discover the minimum point of the area, but it is still difficult to do global converge to minimum point. The global minimum point of $f_{3}$ is $x_{i}=0(i=1,2, \ldots \mathrm{n})$, and the function has a number of local minima hugged. Rastrigin function $f_{4}$ is $x_{i}=0(i=1,2, \ldots \mathrm{n})$, and it can achieve the global minimum of multimodal function.

Operations process simulation tests conducted to obtain space dimensionality: $D=30$, Number groups: $N=20$, Maximum number of iterations: $I m a x=2000$, set the random number test to 100 . Finally, the optimumt results obtained with conventional standard algorithm for the comparative analysis, the results shown in Table 1.

Table 1. Function $\boldsymbol{f}_{1}-\boldsymbol{f}_{4}$ test results

\begin{tabular}{cccc}
\hline & PSO & GA & TSPSO \\
\hline$f_{1}$ & $2.06 \mathrm{E}-014$ & 0.0044 & 0.000 \\
$f_{2}$ & 151.715 & 199.698 & 0.810 \\
$f_{3}$ & 0.0186 & 888.968 & 0.000 \\
$f_{4}$ & 50.001 & 49.487 & 0.000 \\
\hline
\end{tabular}


The results in Table 1 show that iteration of the same number, as well as the same search space area: For function $f_{1}$, PSO algorithm optimization ability and convergence results better than GA algorithm, and the performance of TSPSO algorithm presented is strong on PSO algorithm. Performance comparison of $f_{2}$, GA and PSO algorithm for function is similar. Global Optimization has a big gap, TSPSO algorithm with respect to GA and standard PSO algorithm and its accuracy has been improving, rather approximate global optimal value. Through the comparison of optimization results we can see performance is considerably improved optimization algorithm, mainly because of the disadvantages of improved tabu search algorithm, that is relatively dependent initial solution, combined with excellent climbing ability of the algorithm so that it can eventually obtain results very close to the global optimal solutions of optimization.

Figure 2 to figure 5 for improving the optimization algorithm with the standard PSO test results comparison chart.

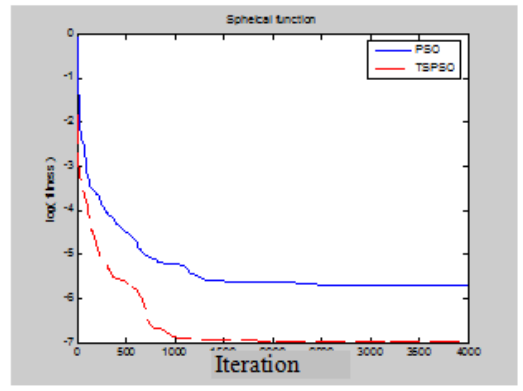

(a)

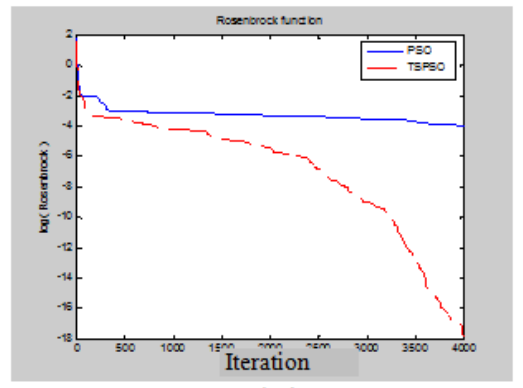

(b)

\section{Figure 2. Spherical function test Figure 3. Rosenbrock function test curve for TSPSO and PSO curve for TSPSO and PSO}

Figure 2 and Figure 3 show the improved algorithm can get the solution to converge to the optimal solution, higher accuracy. Especially for Rosenbrock function, the advantages of the improved algorithm is more outstanding.

Figure 2-5, compared with the standard PSO algorithm, improved PSO algorithm to four test function test results have shown the average convergence speed, algebra substantially reduced, and accuracy greatly improved properties. The $f_{1}$ and $f_{2}$ and $f_{4}$ to test the final results with the theoretical minimum gap are very small, reflecting the improved algorithm of good optimization capability.

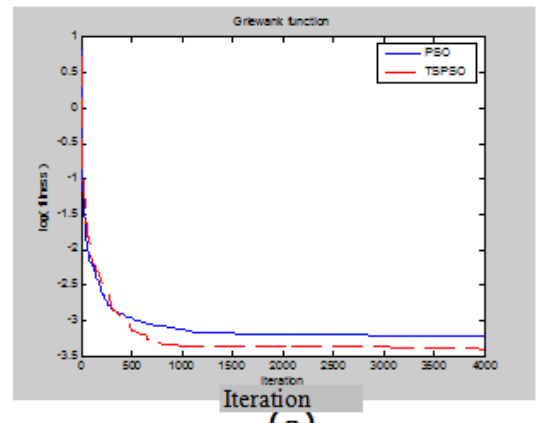

(a)

Figure 4. Griewank function test curve for TSPSO and PSO

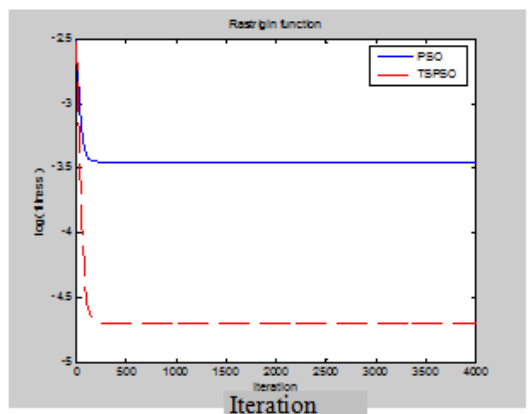

(b)

Figure 5. Rastrigin function test curve for TSPSO and PSO 
Schaffer function $f_{5}$ is a multimodal function. The function characterized by strong shocks, making it difficult for traditional algorithms search for optimal solutions. So for this particular situation, we thought when seeks once the optimal solution is smaller than -0.9999 , then determines as the success, we are here to determine the merits of the standard algorithm for the 100 randomized experiments to search for the number of successes. Evolving algebra the maximum value are the 200, 300, and 500. Table 2 is the standard particle swarm optimization, genetic algorithm, and improvement algorithm computed result.

Table 2. Schaffer function success search number of times

\begin{tabular}{cccc}
\hline A I gebra & PSO & GA & TSPSO \\
\hline 200 & 10 & 2 & 98 \\
\hline 300 & 28 & 10 & 100 \\
500 & 32 & 7 & 100 \\
\hline
\end{tabular}

As shown in Table 2, TSPSO algorithms for search in the number is far greater than a set of particle swarm optimization algorithm and genetic algorithm, and when a sufficiently large number of iterations later, searches can be almost achieving a success rate of $100 \%$.

Simulation results show that presented an improved optimization algorithm, made up for the original typical PSO algorithm for searching for local optimal solutions that are power is relatively weak and prone to premature maturation of disadvantages, a tabu search algorithm for strong "mountain climbing" capacity, speed up the convergence timeand the effectiveness of converged solution, to achieve a combination of complementary advantages.

\section{Distributed Generations Allocation}

\subsection{Mathematical model}

3.1.1 The objective function: Distributed generations are connected into the distribution network which will cause the power flow to be changed, generally the power flow in branches can be reduced, so network loss will reduce. However, if the distributed power capacity is too high, the network is easy to increase the loss. Considering the importance of the power loss, in this paper we select it as the objective function as follows:

$$
\min f=\min P_{\text {Loss }}=\ddot{\sum} G_{k}(i, j)\left[U_{i}^{2}+U_{j}^{2}-2 U_{i} U_{j} \cos \left(\theta_{i}-\theta_{j}\right)\right]
$$

Where, $\mathrm{P}_{\mathrm{Loss}}$ is the power loss value, $G_{k}(i, j)$ is the conductivity in the line $i j, \theta_{\mathrm{i}}$ is the voltage phase angle at node $i, U_{i}$ and $U_{j}$ are the voltage at the node $i$ and node $j$ respectively, $N$ is the total number of branches.

\subsubsection{The constraints of the model}

a) Equality constraints:

$$
h(x)=\left\{\begin{array}{l}
P_{G i}-U_{i} \sum_{j=1}^{N} U_{j}\left(G_{i j} \cos \theta_{i j}+B_{i j} \sin \theta_{i j}\right)=0 \\
Q_{G i}-U_{i} \sum_{j=1}^{N} U_{j}\left(G_{i j} \sin \theta_{i j}+B_{i j} \sin \theta_{i j}\right)=0
\end{array}\right.
$$


Where, $P_{G i}$ is the active power which injects into the node i, $Q_{G i}$ is the reactive power which injects into the node $i, U_{i}$ and $U_{j}$ is the voltage of the position of node $i, \theta_{i}, \theta_{j}$ is the voltage phase angle at node $i$ and $j, G,(i, j)$ is the conductivity in each branch, $B_{i j}$ is the susceptance in the line $i j$.

b) Inequality constraints for the node flow equations:

$$
g(x)=\left\{\begin{array}{c}
U_{\min } \leq U \leq U_{\max } \\
\left|P_{l}\right| \leq\left|P_{l \text { max }}\right| \\
\left|P_{D G, \min } \leq P_{D G} \leq P_{D G, \max }\right| \\
\max \left\{P_{D G, i}\right\} \leq P_{s, \max }-P_{s} \\
I_{s c L} \leq I_{s c L, \text { max }}
\end{array}\right.
$$

Where, $U$ and $P_{l}$ are the voltage value of each node and active power vector value, $P_{D G}$ is the rated capacity of distributed power, $P_{s}$ and $P_{s m a x}$ are the actual power and the maximum power value and the upper limit of power in the system, $I_{s c L}$ and $I_{s c L m a x}$ are maximum shortcircuit current value and the upper limit of breaking current.

\subsection{Numerical example of power system with distributed power network}

3.2.1 The selection of model parameters: The proposed method is applied to the IEEE-33 node system, and verified by simulation using MATLAB 7.6. IEEE-33 node network structure is shown below. In this system, node 0 is as the balance node and the reference power is 100MVA, and the maximum power limit is $600 \mathrm{~kW}$, population size is 100 , maximum number of iterations is 400 .

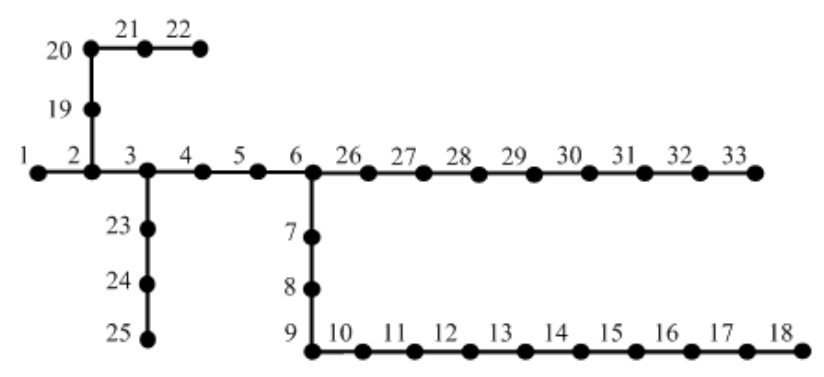

Figure 6. IEEE-33 nodes system

3.2.2 Numerical results analysis: In this power system, firstly, this research analyzes the system operational status which is without DG. Respectively, and adding a distributed power supply and two cases were compared.

Case 0: None DG is connected, then get the system power loss;

Case 1: 1 DG is connected in the system, and analysis to obtain the position and capacity of it, then get the power loss.

Case 2: 2 DGs are connected in the system, then calculate their locations, capacity and the power loss.

Case 3: 3 DGs are connected in the system, then calculate their locations, capacity and analysis of the situation in the system. 
Case 4: 4 DGs are connected in the system, then analysis to obtain the positions and capacity of them, and calculate the power loss.

Table 3. Performance analyze

\begin{tabular}{|c|c|c|c|}
\hline Num. of DGs & Node number for DGs & Capacity for DGs (kW) & Power loss (kW) \\
\hline 0 & - & - & 173.8564 \\
\hline 1 & 12 & 446.2256 & 84.7307 \\
\hline 2 & 24 & 363.4832 & 31.3753 \\
\hline
\end{tabular}

Table 3 shows that using the proposed method in the system with distributed generations, the system power losses declined. Through the resulting data can be seen, case 1 (1 DG connected) is compared with the case 0 (without DG), the power loss reduced $51.26 \%$. And in case 2 , the power loss decreases 81.95 percent, then calculating the voltage value of each point.

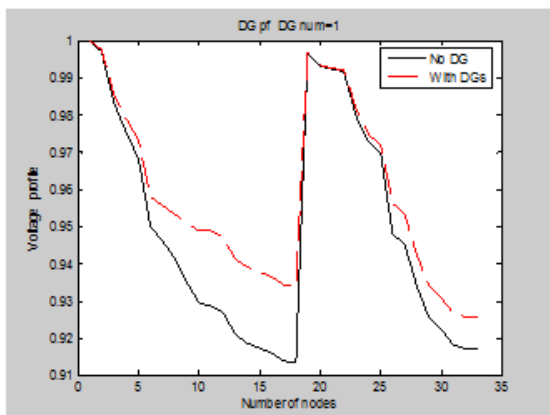

(a)

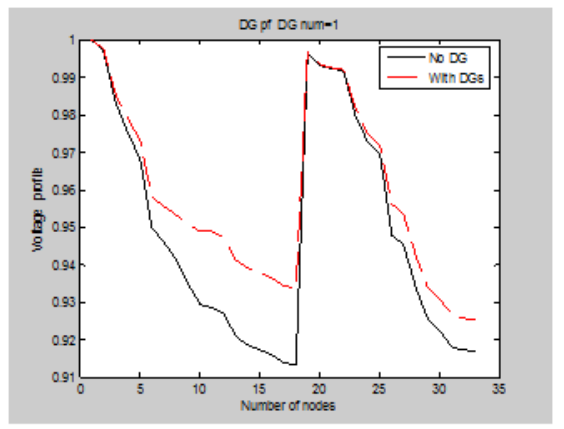

(b)

Figure 7 .Voltage profile changes of each node in case 1 and case 2

Shown in Figure 7, we can get different changes from different DGs. In the power system which with no DG, the voltage value of every node show in Fig.6. Compared with the case 0, the case 2 can obtain more stable voltage values.

From the data above, we can find that using the proposed improved optimization algorithm to optimize the allocation of power generations, not only the power losses had significantly reduced, but voltage stability also obtained a marked decrease. Therefore, this method can prove the feasibility and effectiveness.

Table 4. Performance analyze in the case 3 and case 4

\begin{tabular}{|c|c|c|c|}
\hline Num. of DGs & Node number for DGs & Capacity for DGs (kW) & Power loss (kW) \\
\hline \multirow{3}{*}{3} & 32 & 597.9557 & 31.4047 \\
& 17 & 579.9480 & \\
\hline \multirow{3}{*}{4} & 2 & 329.1353 & \\
& 14 & 477.2567 & 31.3944 \\
& 5 & 455.2771 & \\
\hline
\end{tabular}


The following conclusions can be drawn from the above table, system power losses based on connected number and location of distributed power will change, if the optimization is reasonable, then the power loss can be effectively reduced. As shown in Table 4, according the case 4 , connecting 4 DGs into the system, then the power loss ratio decreased 0.3 percent to $31.3944 \mathrm{~kW}$ than the situation that connecting 3 DGs.

Next, determine the voltage profile. After settlement procedures, the voltage value of each node obtained. The results are showed in Figure 8.

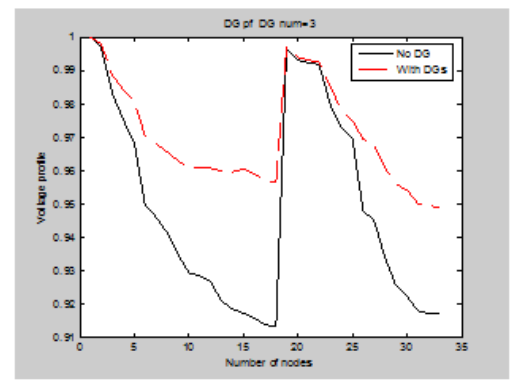

(a)

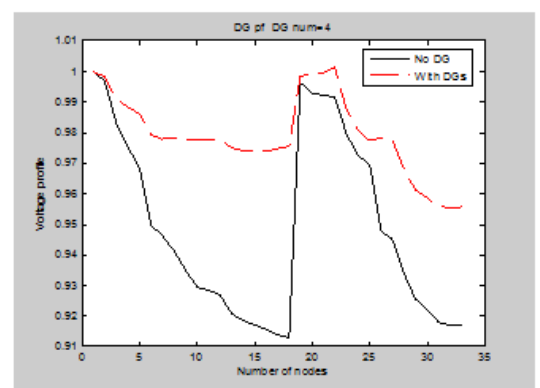

(b)

Figure 8. Voltage profile changes of each node in case 3and case 4

From the Figure 8 can be found: in case 3 and case 4, the system node voltage is gradually stabilized, so the superiority of this method is verified.

The simulation results show that the improved PSO algorithm can be used to achieve optimal allocation by locating and sizing for the system with DG. The proposed algorithm compared the two situations that has or hasn't connect distributed generations. Then finish the simulation and computing in the number of 1,2,3,4 which all the optimized position and capacity of the distributed power can be obtained. Bur when the number of access DG was set an upper limit, for example, the simulation result shows that if the limit number was set as 4 , we can get the optimal solution to keep the system with minimum losses when the selected DG number is 4 under the guidance of the objective function.

Using the method to optimize allocation of the system, we can obtain the minimum loss of the network and the improved node voltage stability of the system. Therefore, the rationality and superiority of the proposed method can be proved by the simulations.

\section{Conclusion}

The development and research of distributed generation technology has get high attention in the field of electric power technology nowadays. But the planning and research for distributed generation connected to the power distribution network has not reached maturity stage now. In this paper, the study of locating and sizing was finished through the improved PSO algorithm.

First, Tabu Search Algorithm was used to improve the PSO algorithm. By making use of both advantages, the speed of optimization and the optimization ability of algorithm were improved. Finally the simulation was finished by the test function, then the result proved the rationality and other related advantages of the algorithm. In addition, the distributed generation of distributed power network was optimal allocated. The main content of the work was to determine the access number, location and capacity of distributed generation according to the result of power flow calculation, which was obtained through the improved PSO 
algorithm with given objective function. Then realize to select the optimization scheme and test the superiority by simulation.

\section{References}

[1] M. Zangiabadi, R. Feuillet and H. Lesani, "An Approach to Deterministic and Stochastic Evaluation of the Uncertainties in Distributed Generation Systems", In Proceedings of 20th International Conference on Electricity Distribution, (2009) June 8-11: Prague, Czech republic.

[2] H. Yassami, A. Moeini and S. M. R. Rafiei, "Optimal distributed generation planning considering reliability, cost of energy and power loss", Scientific Research and Essays, vol. 6, no. 9, (2011).

[3] M. Mohammadi and M. A. Nasab, "DG Placement with Considering Reliability Improvement and Power Loss Reduction with GA Method", Research Journal of Applied Sciences, Engineering and Technology, vol. 8, no. 3, (2011).

[4] M. Lalitha, V. Reddy and N. Reddy, "DG source allocation by fuzzy and clonal selection algorithm for minimum loss in distribution system", Distributed Generation and Alternative Energy Journal, vol. 4, no. 26, (2011).

[5] P. Paliwal and N. P. Patidar, "Distributed Generator Placement for Loss Reduction and Improvement in Reliability", World Academy of Science, Engineering and Technology, vol. 10, no. 45, (2010).

[6] A. Rezazadeh, M. Sedighizadeh and A. Alavian, "Optimal sizing and placement of distributed generation in a network system”, International Journal of Electrical Power and Energy Systems, vol. 8, no. 32, (2010).

[7] E. A. Belati, "Transmission loss allocation based on optimal power flow and sensitivity analysis", International Journal of Electrical Power and Energy Systems, vol. 4, no. 30. (2008).

[8] E. V. Wirmond, "TCPST allocation using optimal power flow and Genetic Algorithms", International Journal of Electrical Power and Energy Systems, vol. 4, no. 33, (2011).

[9] P. Álvarez, "Development of a measure model for optimal planning of maintenance and improvement of roads", Computers and Industrial Engineering, vol. 3, no. 52, (2007).

[10] P. Reche-López, "Comparison of metaheuristic techniques to determine optimal placement of biomass power plants", Energy Conversion and Management, vol. 8, no. 50, (2009).

[11] H. Mori, "Development of Hybrid-Coded EPSO for Optimal Allocation of FACTS Devices in Uncertain Smart Grids", Procedia Computer Science, vol. 6, (2011).

[12] A. C. Rueda-Medina, "A mixed-integer linear programming approach for optimal type, size and allocation of distributed generation in radial distribution systems", Electric Power Systems Research, vol. 1, no. 97, (2012). 